\title{
Fabricación de nanoestructuras por ablación láser y su uso en SERS con bajo umbral de detección: el caso de Ag-Nps ${ }^{\diamond}$
}

\section{Manufacturing of nanoestructures by laser ablation and its use in SERS with low detection threshold: the case of Ag-NPs}

\author{
V. Alonso Camarena-Chávez, ${ }^{*}$ H. Mauricio Reynoso De La Cruz, ${ }^{*}$ \\ J. Ulises Álvarez-Martínez,* Alejandro Martínez-Bórquez,* \\ G. Gutiérrez-Juárez, G. Ramos-Ortiz, ** R. Castro-Beltrán*,॰
}

\begin{abstract}
In this work we present the manufacture of silver nanoparticles (Ag-NPs) by means of laser ablation (AL) in aqueous medium, and its application as elements of Raman signal enhancement through SERS type processes. This methodology allows to obtain stable suspensions of NPs free of the use of chemical processes, with dispersions of relatively small sizes and of easy processing for the implementation of SERS substrates of silica. Through the use of aliquots at different concentrations of Rhodamine $6 \mathrm{G}$ (R6G), it is demonstrated that with these Ag-NPs, Raman signal detection thresholds are reached in concentrations of the order of $\mathrm{nM}$. The study of the distribution of Ag-NPs on a silica substrate was carried out by atomic force microscopy (AFM). In turn, some simulation results of the magnitude of the fields are generating hot spot in specific regions of the Ag-NPs taking into account the morphology of some of the agglomerates identified in the electron scanning microscopy (SEM) in the SERS substrates. In comparison to some previous reports, the present work demonstrates low detection thresholds and simple implementation in SERS substrates.
\end{abstract}

KEYWORDS: laser ablation, silver nanoparticles, SERS.

RESUMEN: En este trabajo presentamos la fabricación de nanopartículas de plata (Ag-NPs) por medio de ablación láser ( $\mathrm{AL}$ ) en medio acuoso, y su aplicación como elementos de realce de señales Raman a través de procesos tipo SERS. Esta metodología permite obtener suspensiones estables de NPs libres del uso de procesos químicos, con dispersiones de tamaños relativamente pequeñas y de fácil procesamiento para la implementación de sustratos SERS de sílice. A través del uso de alícuotas a diferentes concentraciones de Rodamina $6 \mathrm{G}(\mathrm{R} 6 \mathrm{G})$, se demuestra

Recibido: 2 de septiembre de 2019.

Aceptado: 22 de octubre de 2019.

$\checkmark$ Esta investigación fue parcialmente apoyada por el Consejo Nacional de Ciencia y TecnologíaMéxico (Fronteras de la Ciencia-2016, Proyecto No.2029 y proyecto 291434) y por la Dirección de Apoyo a la Investigación y el Posgrado (DAIP) de la Universidad de Guanajuato (Proyectos CIIC: 341/2019, CIIC: 36/2019).

* Universidad de Guanajuato, División de Ciencias e Ingenierías, Departamento de Ingeniería Física, Cuerpo Académico de Mecánica Estadística. Loma del Bosque \#103, Lomas del Campestre, 37150 León, Gto.

** Centro de Investigaciones en Óptica A. C., A. P. 1-948, 37000 León, Gto., México.

- Autor de correspondencia: cbrigoberto@fisica.ugto.mx 
que con estas Ag-NPs se alcanzan umbrales de detección de señal Raman en concentraciones del orden de nM. El estudio de la distribución de las Ag-NPs sobre un sustrato de sílice se llevó a cabo por microscopía de fuerza atómica (AFM). A su vez, se presentan algunos resultados de simulación de la magnitud de los campos generando hot spot en regiones específicas de las Ag-NPs tomando en cuenta la morfología de algunos de los aglomerados identificados por microscopía de barrido electrónico (SEM) en los sustratos SERS. En comparación con algunos reportes previos, el presente trabajo demuestra bajo umbral de detección e implementación sencilla en sustratos SERS.

PALABRAS CLAVE: ablación láser, nanopartículas de plata, SERS.

\section{Introducción}

El efecto de esparcimiento Raman aumentado por superficie (SERS) fue reportado por primera vez a mediados de la década de los años 70 del siglo XX (Fleischmann et al., 1974). Los estudios de SERS de diferentes compuestos orgánicos arrojan principalmente la información de la huella digital molecular del compuesto, a través de un espectro característico con múltiples picos soportados en el mismo. La importancia de estos picos es que dan información de la estructura molecular del compuesto, por ejemplo, de sus estados vibracionales y rotacionales (Fleischmann et al., 1974; Dedic et al., 2017). Los inconvenientes principales de esta técnica espectroscópica provienen directamente de una condición inherente en la amplitud de la señal Raman, la cual es proporcional a $10^{-10}$ veces más baja que la intensidad de la luz láser de excitación (Kumar Sur, 2017). Como ya mencionamos, en la década de los años 70 del siglo XX, Fleischmann y colaboradores de la Universidad de Southampton, Reino Unido, reportaron señales Raman amplificadas por un factor de 1 millón, al llevar a cabo estudios sobre compuestos orgánicos depositados directamente en electrodos de plata rugosos (Fleischmann et al., 1974; Kumar Sur, 2017). Este descubrimiento marcó la era de esta técnica espectroscópica originando el desarrollo de nuevas teorías, nuevos protocolos de caracterización espectroscópica de materiales y la oportunidad de potencializar las aplicaciones de sistemas metálicos nanoestructurados. En este sentido y considerando que el factor de realce está fuertemente ligado con las características de la superficie metálica, por caso, el tipo de metal, su forma e interacción híbrida con el compuesto orgánico adsorbido en su superficie, es que la ingeniería de materiales metálicos nanoestructurados, nanotecnología, ofrece ventajas extraordinarias para la innovación en técnicas espectroscópicas. Algunos de los aspectos más importantes en nanotecnología son las oportunidades diversas en los protocolos de fabricación de dichos materiales nanoestructurados. Gran parte del auge en esta rama científica se debe a que a través de diversos métodos (físicoquímicos, fotoquímicos, por evaporación/condensación, libres de químicos "verdes", etc.) es posible sintetizar nanoestructuras tan simples como esferas, o, complejas, como nanotubos, nanopirámides, entre otras más. La importancia en el control de la forma y el tamaño radica en las nuevas oportunidades tecnológicas que re- 
presentan, por ejemplo, en el desarrollo de nanoantenas, aplicaciones en microláseres, aplicaciones en celdas solares y nuevas oportunidades espectroscópicas como SERS (Castro-Beltrán et al., 2017; Shi et al., 2013). Para el caso particular de estudios basados en SERS, la forma de las NPs, su tamaño y su capacidad bioquímica superficial para contener (adsorber) compuestos orgánicos en su superficie es fundamental, así como el realce del campo eléctrico en su superficie (Yingcheng et al., 2015; Yang et al., 2014). Por ejemplo, la interacción del campo eléctrico con una nanopartícula (en su representación 2D) de forma esférica puede resultar en diferentes condiciones de soporte del campo en la superficie. La figura 1 presenta una simulación de la distribución del campo eléctrico para NPs esféricas de radio de $50 \mathrm{~nm}$ en donde la radiación óptica corresponde a una onda plana incidiendo en la dirección $\mathrm{X}$. Esta geometría y dimensiones de NPs son las que típicamente se obtienen con la técnica de ablación láser de metales nobles presentada en este trabajo. Las simulaciones fueron llevadas a cabo considerando la longitud de onda de resonancia para NPs de estas dimensiones y la longitud de trabajo del equipo Raman con que se cuenta.

De la figura 1 notamos regiones con máxima distribución del campo en la superficie de la esfera. Se observa también, que a la longitud de onda cer-

FIGURA 1. Distribución del campo eléctrico en NPs de geometría esférica a dos longitudes de incidencia, 390 y $780 \mathrm{~nm}$, mostrados en panel superior e inferior, respectivamente.
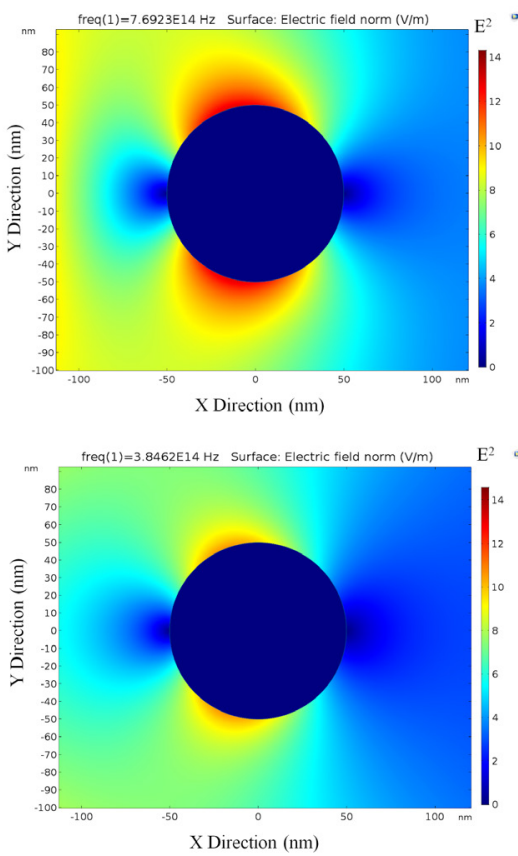

Fuente: Elaboración de los autores.
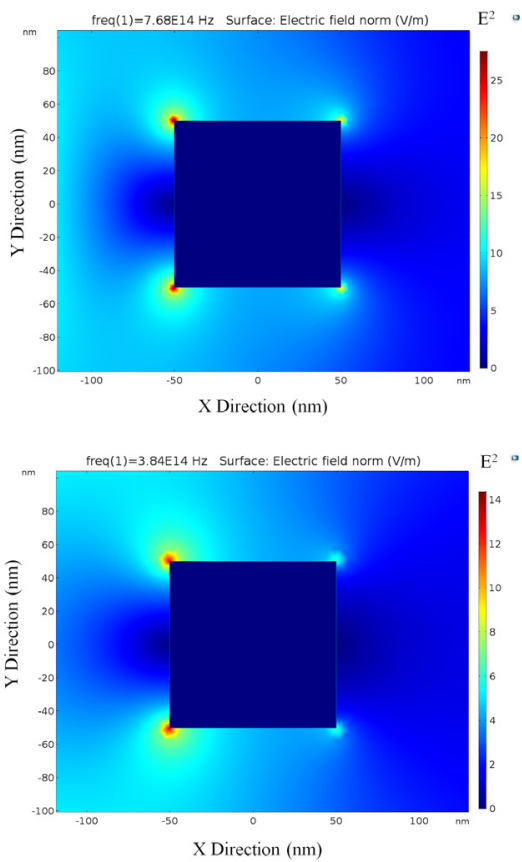
cana a la de resonancia (390 $\mathrm{nm}$ ) el campo alcanza mayor intensidad en la superficie que para una longitud de onda $(780 \mathrm{~nm}$ ) más lejana a la de resonancia. Aunque para efectos de este trabajo el interés se centra en geometrías esféricas, debe mencionarse que, aunque en menor proporción, la técnica de ablación láser también produce NPs de formas irregulares, con superficies incluso puntiagudas que soportan efectos de campo locales conocidos como hot spot (Kumar Sur, 2017) El efecto de distribución local del campo en la superficie de las NPs da lugar al efecto de realce (EF) de señal Raman. El EF está definido por EF = (ISERS X NNORMAL)/(IRAMAN X NSERS) donde ISERS y IRAMAN son, respectivamente, las intensidades de la señal Raman en su configuración aumentado por superficie y normal mientras que, NNORMAL y NSERS son el número de moléculas probadas en su configuración normal y aumentado, respectivamente. Finalmente, se menciona que en un tratamiento más formal sobre el efecto SERS, se debe considerar que las NPs metálicas soportan plasmopolaritones localizados en su superficie, existiendo un acoplamiento eficiente electromagnético con materiales circundantes. En este enfoque teórico, el realce de señal Raman está determinado por el factor de Purcell que relaciona el factor de calidad $Q$ y el volumen específico $V_{\text {eff }}$ de los plasmopolaritones localizados.

Los protocolos de síntesis de NPs metálicas también han sido de interés para la comunidad científica, sobre todo cuando se busca que sean simples, de bajo costo, que produzcan en masa y que estos den como resultados partículas estables por tiempos muy largos (meses), biocompatibles (para el caso de aplicaciones biomédicas) y con una distribución homogénea en cierto volumen. Debido al enfoque del presente trabajo, la síntesis de las AgNPs se llevó a cabo a partir de protocolos, los cuales pueden catalogarse dentro de la categoría de procesos verdes, esto es, libres de contaminantes químicos (Rosales et al., 2018). Previo a este trabajo, se ha reportado la fabricación de Ag-NPs por métodos de ablación láser con el propósito de ser usadas en aplicaciones SERS. Así, por ejemplo, se ha reportado el depósito de Ag-NPs en sustratos inmersos en ambiente de Ar durante el proceso de ablación; si bien con dicha técnica se pueden controlar hasta cierta medida las dimensiones de las NPs obtenidas, tiene como desventaja requerir de un ambiente atmosférico controlado (D’Andrea et al., 2009) La producción de Ag-NPs por ablación láser en líquido y su deposición en sustratos SERS por medio de la técnica electrohidrodinámicas ha sido reportada (Nebogatikov et al., 2015). Se señala que en estos dos casos citados se usó como analito de prueba SERS el colorante rodamina 6G. En el presente trabajo presentamos la generación de suspensiones acuosas de NPs estables por el método de ablación láser. A diferencia de los métodos reportados en las referencias (D’Andrea et al., 2006; Nebogatikov et al., 2015) que implican procesos complejos de deposición de las NP's en sustratos SERS, en nuestro caso demostramos que de manera sencilla y directa se pueden obtener sustratos SERS sin sacrificar los umbrales de detección de analitos. 


\section{Protocolo de fabricación por procesos de ablación láser}

La figura 2 muestra el arreglo experimental utilizado para la síntesis de Ag-NPs a partir de procesos de ablación láser. El sistema utiliza un láser pulsado de alta potencia Nd:YAG con un ancho temporal del pulso de $8 \mathrm{~ns}$, con una frecuencia de repetición de $10 \mathrm{~Hz}$ y una longitud de onda de trabajo centrada en $532 \mathrm{~nm}$.

El láser es dirigido y enfocado en la oblea de plata a partir de un sistema óptico que asegura alineación y una energía de trabajo de $680 \mathrm{~mJ} \mathrm{~cm}{ }^{-2}$. La oblea de plata con un espesor aproximado de 400 um y $0.6 \mathrm{~cm}$ de diámetro es adherida a un portaobjetos $y$, por último, sumergida en un frasco de vidrio con $4 \mathrm{~mL}$ de agua destilada. El sistema óptico utilizado en conjunto con el volumen de agua del vaso de vidrio, la posición de la oblea en el fondo del mismo y el espesor de la misma son las adecuadas para que los pulsos de luz láser impacten directamente en la superficie de la oblea, ablacionando su superficie.

Previo al proceso de ablación, la oblea adherida a un portaobjetos se lija y limpia meticulosamente con la finalidad de eliminar cualquier exceso de pegamento que pueda quedar sobre su superficie. La limpieza de este se lleva a cabo con agua destilada con aproximadamente 3 o 4 ciclos de repetición, el uso de solventes en este punto puede dañar la adherencia de la oblea a la superficie de vidrio y propiciar el desprendimiento de la oblea durante la ablación.

En el transcurso del proceso de ablación, se toman diferentes espectros de absorción UV-vis como un sistema de control en el proceso de síntesis (figura 2). La evolución del espectro de absorción nos muestra que la síntesis de AgNPs se presenta desde los primeros 10 minutos (Ag10) del proceso de fabricación. Conforme la muestra continúa expuesta a excitación láser (Ag10-Ag20), el ancho en el espectro se va haciendo más angosto y la intensidad del mismo

FIGURA 2. Representación artística del arreglo experimental utilizado para la síntesis de Ag-NPs por procesos de ablación láser directa. El sistema utiliza un láser pulsado Nd:YAG centrado con un longitud de onda de trabajo a $532 \mathrm{~nm}$. Se muestran fotografías de la oblea sumergida en el recipiente de vidrio y la solución coloidal final, así como la evolución en el espectro de absorción durante todo el proceso de síntesis. Los subíndices en $\mathrm{Ag}_{10}, \mathrm{Ag}_{15}$ y $\mathrm{Ag}_{15}$ denotan el tiempo de ablación en minutos.

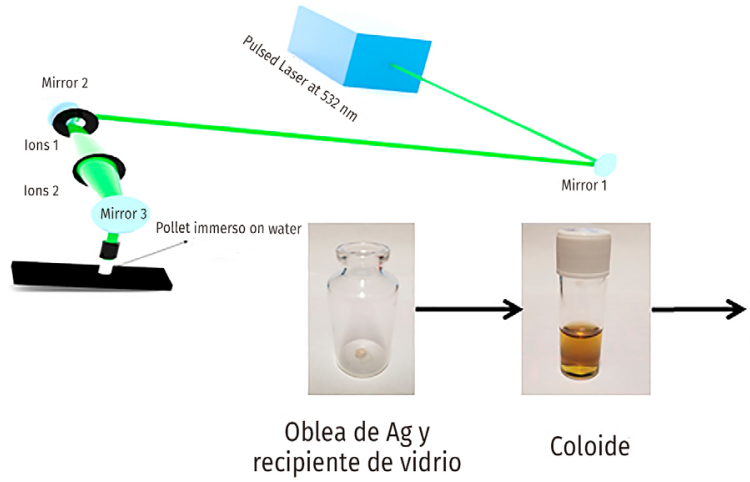

Fuente: Elaboración de los autores.

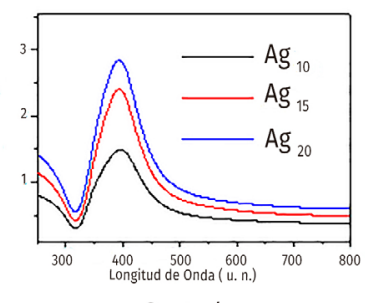

Control 
crece. Esto atiende a que la síntesis de las nanopartículas es más homogénea en tamaño y la concentración de las mismas también crece. Para estimar la concentración final en $\mathrm{Mol} \mathrm{L}^{-1}$ de la solución coloidal es necesario saber los pesos y volúmenes de todos los componentes utilizados en los procesos de fabricación. La tabla 1 muestra un ejemplo de estimación de la concentración a (Ag15).

\section{Preparación del sustrato para SERS, AFM, SEM y COMSOL}

Para la preparación del sustrato para la caracterización SERS se llevó a cabo el siguiente procedimiento: las Ag-NPs fueron depositadas sobre portaobjetos comunes. Previo a esto, los portaobjetos se someten a un procedimiento de limpieza donde se limpian con alcohol isopropílico y secados con aire comprimido para eliminar cualquier residuo de polvo. Finalmente, se sometieron a una temperatura de $200{ }^{\circ} \mathrm{C}$ para deshidratarlos y eliminar cualquier residuo del solvente. Una vez limpios, se depositó sobre ellos un volumen de $70 \mu \mathrm{l}$ de nanopartículas de plata. Las nanopartículas de plata se depositaron con una micropipeta graduada en $2.5 \mu \mathrm{l}$ depositando un volumen total de 10 $\mu \mathrm{l}$ de una sola vez y se esperó a que la gota secara. El proceso se repitió de la misma manera hasta llegar al volumen total de $70 \mu \mathrm{l}$. Se siguió este procedimiento para evitar que la mayoría de las nanopartículas se depositarán en el perímetro de la gota, logrando una distribución más homogénea sobre el sustrato. Una vez depositadas las nanopartículas se depositó un volumen de $10 \mu \mathrm{l}$ de rodamina en concentraciones que iban de $10^{-3}$ a $10^{-8}$ molar.

Con la finalidad de conocer cómo se organizan las Ag-NPs depositadas sobre el sustrato de vidrio tomando en cuenta el procedimiento de depósito descrito arriba, se llevaron a cabo estudios de microscopía de fuerza atómica (figura 3).

En la figura 3 se presenta una imagen de las nanopartículas tomada por microscopio, depositadas sobre un sustrato de vidrio, y el estudio de AFM para ver con mayor detalle la distribución que tienen estas sobre el sustrato. Se observa que las nanopartículas tienden a aglomerarse y a organizarse por regiones proporcionales a varios micrómetros. El RMS, que calculado para la región resaltada fue $69.67 \mathrm{~nm}$, indica que la capa Ag-NPs es muy uniforme. En la gráfica del AFM se observa la aglomeración de las partículas, lo cual indica que la forma de depósito puede ser adecuada pues hay regiones donde la rodamina

TABLA 1. Detalles de las masas medidas para la oblea de plata antes y después del proceso de ablación láser y que son usadas para calcular la concentración final de las muestras coloidales. Donde: moles = masa $/$ masa atómica, masa = masa inicial-masa final y la molaridad = moles $/ \mathrm{L}$ solución .

\begin{tabular}{|c|c|c|c|c|c|c|c|}
\hline Muestra & $\begin{array}{c}\text { Oblea } \\
(\mathrm{g})\end{array}$ & $\begin{array}{c}\text { Agua } \\
(\mathrm{g})\end{array}$ & $\begin{array}{c}\text { Oblea } \\
\text { ablacionada } \\
(\mathrm{g})\end{array}$ & $\begin{array}{c}\text { Coloide } \\
(\mathrm{g})\end{array}$ & $\Delta \mathrm{g}$ & $\begin{array}{c}\text { Moles } \\
(\mathrm{M}) \\
\mathrm{E}-05\end{array}$ & $\begin{array}{c}\mathrm{M} \\
(\mathrm{mol} / \mathrm{L}) \\
\mathrm{E}-03\end{array}$ \\
\hline Ag15 & 766.2 & 4081.6 & 765.0 & 3963.3 & 1.2 & 1.11 & 2.8 \\
\hline
\end{tabular}

Fuente: Elaboración de los autores. 
FIGURA 3. Estudio AFM de la distribución de las nanopartículas sobre el sustrato.

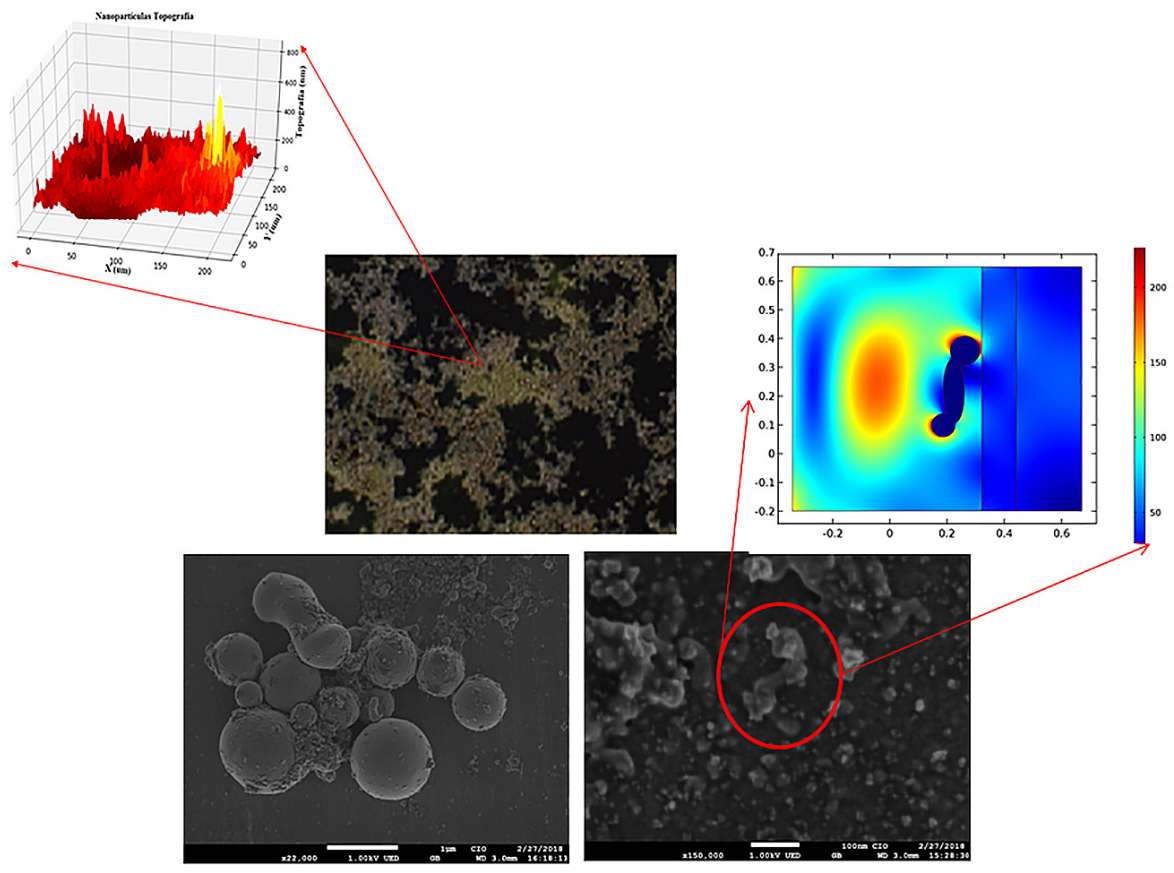

Nota: Imagen tomada con un objetivo de 100X. Imágenes SEM de las Ag-NPs en diferentes regiones del sustrato; en todas se observa la formación de closters La forma de las NPs es completamente esférica. Un ejemplo de la simulación de soporte del campo eléctrico correspondiente a una onda de $780 \mathrm{~nm}$ se muestra también.

Fuente: Elaboración de los autores.

puede adsorberse a las nanopartículas y con esto asegurar la interacción del campo con las mismas y amplificar la señal Raman correspondiente.

La figura 3 muestra, además, un ejemplo en un zoom de la organización de las Ag-NPs en aglomerados. La distribución del campo eléctrico en este aglomerado de Ag-NPs es simulada mediante elemento. Vemos que a 780 $\mathrm{nm}$, que es una longitud de onda de trabajo común en equipo Raman, y que aun y cuando está relativamente lejana de la región de resonancia de las de Ag-NPs, se crean máximos de amplitud del campo del tipo hot spot soportados en el arreglo de Ag-NPs, lo cual asegura el realce de la señal Raman en esas regiones del arreglo cuando dicha longitud de onda es usada. En nuestro proceso, mostramos experimentalmente el caso de otra longitud de onda muy común en equipos Raman, de 633 nm, siendo más cercana a la región de resonancia plasmónica (ca $400 \mathrm{~nm}$ ) de nuestras Ag-NPs denotadas en la figura 2. La excitación del máximo de la banda plasmónica generaría un señal Raman fuertemente realzada, no obstante, se descarta usar un láser con emisión cercana a dicha resonancia por los fuertes efectos de fluorescencia que puede generar en el análisis de analitos. 


\section{Obtención de la señal Raman, procesamiento y resultados}

Para obtener la señal Raman se utilizó un microscopio confocal Raman Witec Alpha 300, con un CCD de 1024 patrones separados a $2.7553 \mathrm{~cm}^{-1}$ aproximadamente; el láser usado como fuente de excitación del proceso fue un helio-neón centrado en $633 \mathrm{~nm}$ con una potencia máxima de $35 \mathrm{~mW}$; para observar las muestras y enfocar el haz sobre estas se usó un objetivo de microscopio de 100X con apertura numérica de 0.9.

La adquisición de los espectros se llevó a cabo considerando un tiempo de integración de 10 segundos, con una acumulación de 5 espectros.

Para el procesamiento de los espectros, primero se resta el ruido obtenido de analizar el sustrato limpio y sin muestra orgánica. Posteriormente, se toman puntos sobre la base del espectro que no pertenezcan a algún pico del espectro (fluorescencia) y se realiza un ajuste polinomial, encontrándose que un polinomio de grado 7 ajusta bien a los datos del espectro. Más adelante, se resta el ajuste a los datos y se obtiene el espectro final. La figura 4 muestra la compilación de todo el análisis desde (superior izquierda) la obtención del espectro original, (superior derecha) el polinomio con el cual se llevarán a cabo los ajustes y, por último (inferior) el resultado del ajuste. Vemos que este procedimiento asegura tanto un tratamiento a las señales adecuado, como la eliminación de los fenómenos de fluorescencia en los que se soportan las señales Raman y, con ello, la identificación de los picos Raman de forma clara.

FIGURA 4. Procedimiento para procesar la señal Raman mostrando el espectro sin procesamiento, con el ajuste polinomial y el espectro final totalmente procesado.
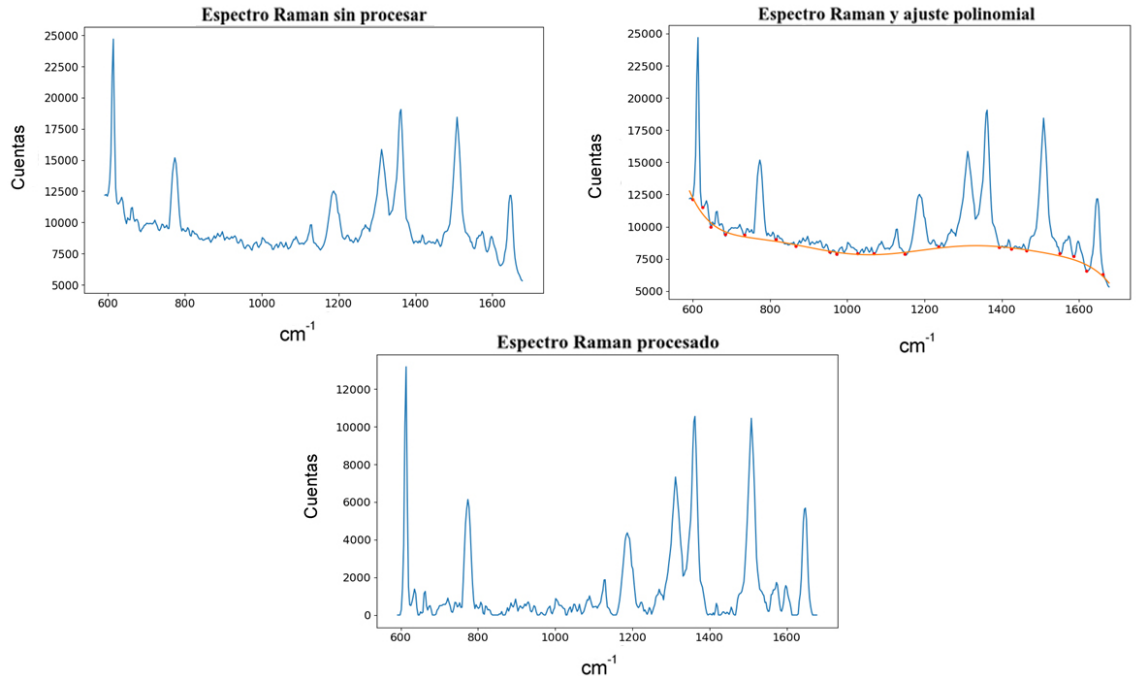

Fuente: Elaboración de los autores. 
Una vez procesados los espectros para eliminar fluorescencia, lo siguiente es identificar las posiciones espectrales de los picos característicos de cada señal. En la figura 5 se observan los picos espectrales característicos de la R6G con este tipo de estudios utilizando Ag-NPs fabricadas por procesos de ablación láser. Al realizar una comparación con los resultados reportados, se encontró que los picos obtenidos (1360, 1510, 1572 y $\left.1650 \mathrm{~cm}^{-1}\right)$ corresponden adecuadamente con los reportados en otros trabajos para la rodamina 6G (He et al., 2012; Škantárová et al., 2013).

FIGURA 5. Espectro Raman con los picos más característicos de la R6G.

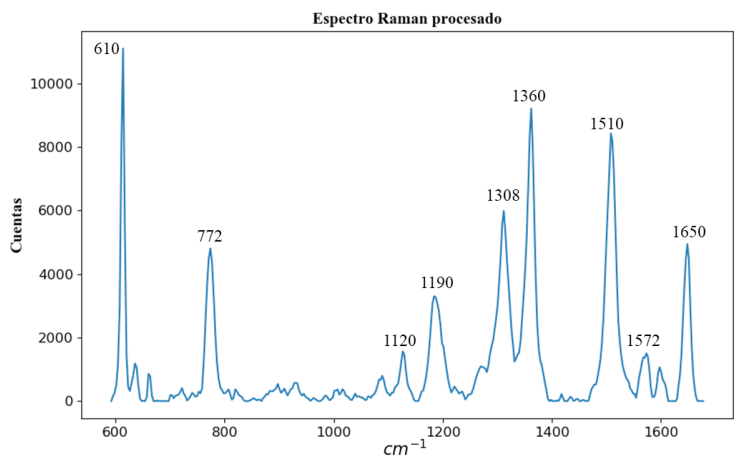

Fuente: Elaboración de los autores.

Con la finalidad de mostrar el umbral de detección en mol/L y la capacidad de la técnica a través de el uso de este tipo de Ag-NPs fabricadas por procesos de ablación láser, la figura 6 muestra la evolución de los espectros SERS a diferentes concentraciones de R6G.

FIGURA 6. Espectros Raman a las distintas concentraciones estudiadas de rodamina $6 \mathrm{G}$ de $10^{-3}$ a $10^{-8}$ $\mathrm{Mol} / \mathrm{L}$.

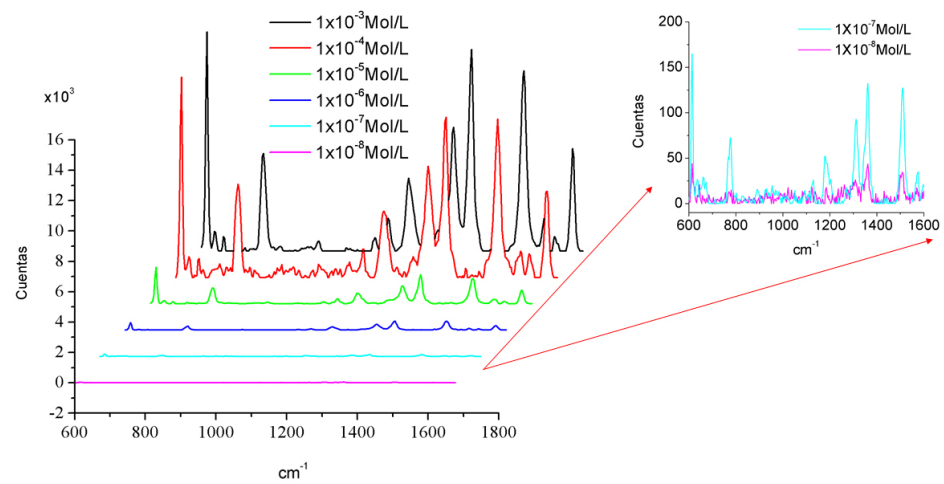

Fuente: Elaboración de los autores. 
Se observa que conforme disminuye la concentración la intensidad del espectro decrece y, además, ciertos picos característicos ya no se encuentran. Son los picos remanentes los que nos permiten determinar que la señal Raman de la R6G está presente a concentraciones muy bajas, sobre todo los picos 1360 y $1510 \mathrm{~cm}^{-1}$ son los que siguen detectando a bajas concentraciones como $10^{-8} \mathrm{~mol} / \mathrm{L}$.

De la figura 6 es posible seguir la evolución en la amplitud de la señal Raman de varios de los picos más característicos de los espectros obtenidos (figura 7). De la figura 7a, se puede concluir que el comportamiento de la intensidad con respecto de la concentración no tiene un comportamiento lineal, que al incrementar la concentración tenemos un realce del espectro, y para los picos en 1190, 1308 y $1572 \mathrm{~cm}^{-1}$ está próxima la saturación. En la figura $7 \mathrm{~b}$, se reporta la fluorescencia a $1000 \mathrm{~cm}^{-1}$ en donde se puede ver un crecimiento monótono, como es de esperarse por el incremento en la concentración.

\section{Conclusiones}

Para terminar, los resultados más sobresalientes respecto al uso de Ag-NPs fabricadas por procesos de ablación láser como elementos de realce de señales Raman han sido presentados en este trabajo. Las Ag-NPs fueron fabricadas siguiendo los protocolos de ablación láser con energías promedio proporciona-

FIGURA 7. a) Intensidad de los espectros Raman para 1190, 1308, 1360 y $1572 \mathrm{~cm}^{-1}$ como función de la concentración de $10^{-3}$ a $10^{-8} \mathrm{~mol} / \mathrm{L}$. b) Intensidad de fluorescencia Raman para $1000 \mathrm{~cm}^{-1} \mathrm{como}$ función de la concentración de $10^{-3}$ a $10^{-8} \mathrm{~mol} / \mathrm{L}$.

a)

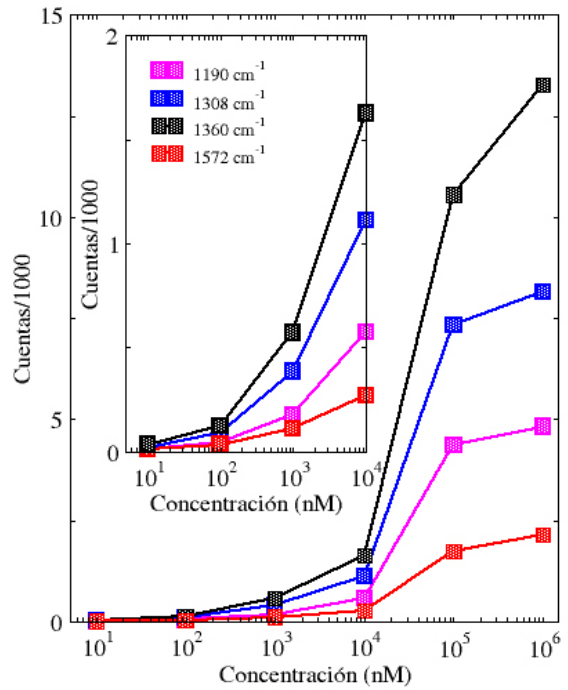

b)

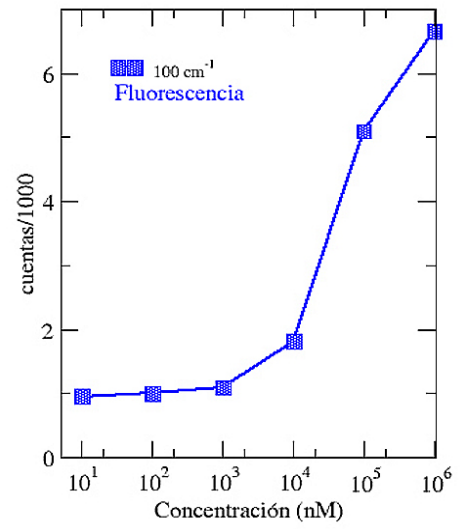

Fuente: Elaboración de los autores. 
les a $650 \mathrm{~mJ} / \mathrm{cm}^{2}$ utilizando un sistema láser Nd:YAG. El seguimiento en el espectro de absorción como medida de control nos permitió observar que a 10 minutos del proceso de fabricación, la presencia de partículas ya era notoria. A 20 minutos de excitación láser contábamos con una solución coloidal con un espectro de absorción característico para Ag-NPs de forma esférica. El umbral característico de detección de señal Raman con estas resultó ser 0.1 nM. A partir de este set de resultados, vemos que la fabricación de Ag-NPs por procesos de ablación láser directa representa oportunidades únicas en términos de métodos alternos de fabricación comparados con los métodos químicos. A su vez, dado que en este proceso de fabricación no es necesario el uso de compuestos químicos, el protocolo y la técnica se catalogan como un proceso libre de sustancias tóxicas "verde" ya que, además, las Ag-NPs fueron sintetizadas directamente en agua destilada, haciéndolas biocompatibles.

\section{Referencias}

Castro-Beltrán, Rigoberto; Vinh M. Diep, Soheil Soltani, Eda Gungor, Andrea M. Armani. (2017). Plasmonically enhanced kerr frequency combs. ACS Photonics, 4: 2828. http://dx.doi.org/10.1021/acsphotonics.7b00808

D’Andrea, C.; F. Neri, P. M. Ossi, N. Santo, S. Trusso. (2009). The controlled pulsed laser deposition of Ag nanoparticle arrays for surface enhanced Raman scattering. Nanotechnology, 20: 245606. http://dx.doi.org/10.1088/0957-4484/20/24/245606

Dedic, Chloe E.; Terrence R. Mayer y James B. Michael (2017). Single-shot ultrafast coherent anti-stokes Raman scattering of vibrational/rotational nonequilibrium. Optica, 4: 563-570. http://dx.doi.org/10.1364/OPTICA.4.000563

Fleischmann, M.; P. J. Hendra y A. J. McQuillan. (1974). Raman spectra of pyridine adsorbed at a silver electrode. Chemical Physics Letters, 26 (2) 163-166.http:// dx.doi.org/10.1016/0009-2614(74)85388-1

He, X. N.; Y. Gao, M. Mahjouri-Samani, P. N. Black, J. Allen, M. Mitchell, W. Xiong, Y. S. Zhou, L. Jiang y Y. F. Lu. (2012). Surface-enhanced Raman spectroscopy using gold-coated horizontally aligned carbon nanotubes. Nanotechnology, 23: 205702. http://dx.doi.org/10.1088/0957-4484/23/20/205702

Kumar Sur, Ujjal. (2017). Surface-enhanced Raman scattering, En Khan Maaz (ed.), Raman spectroscopy and applications, cap. 14. https://www.intechopen.com/ books/raman-spectroscopy-and-applications/surface-enhanced-raman-scattering. http://dx.doi.org/10.5772/66084

Nebogatikov, M. S.; V. Ya. Shur, A. E. Tyurnina, R. V. Kozinr, V. Yu. Sukhanova, E. A. Mingaliev, D. V. Zorikhin. (2015). Surface-enhanced raman scattering using silver nanoparticles produced by laser ablation in liquid. Ferroelectrics, 477: 54-62. https://doi.org/10.1080/00150193.2015.999629

Rosales, J. E. Alba; G. Ramos-Ortiz, G. Martínez-Ponce, R. Castro-Beltrán, L. PoloParada y G. Gutiérrez-Juáreza, (2018). Dual photoacoustic monitoring in laser ablation synthesis of silver nanoparticles to find in situ their fluence threshold 
formation. Results in Physics, 11: 350-357.

http://dx.doi.org/10.1016/j.rinp.2018.08.039

Shi Ce, Soheil Soltani, Andrea M. Armani. (2013). Gold nanorod plasmonic upconversion microlaser. Nano Lett., 13: 5827.

http://dx.doi.org/10.1021/nl4024885

Škantárová, Lenka; Andrej Orinák, Renáta Orináková, Monika Jerigová, Monika Stupavská y Dusan Velicd. (2013). Functional silver nanostructured surfaces applied in SERS and SIMS. Surf. Interface Anal., 45: 1266.

http://dx.doi.org/10.1002/sia.5267

Yang, Jing; Fanghui Ren, Xinyuan Chong, Donglei Fan, Swapnajit Chakravarty, Zheng Wang, Ray T. Chen y Alan X. Wang. (2014). Guided-mode resonance grating with self-assembled silver nanoparticles for surface-enhanced raman scattering spectroscopy. Photonics, 14: 380-389.

http://dx.doi.org/10.3390/photonics1040380

Yingcheng, Pan; Xiaoyu Guo, Jinglu Zhu, Xuan Wang, Han Zhang, Yan Kang, Ting Wu, Yiping Du. (2015). A new SERS substrate based on silver nanoparticle functionalized polymethacrylate monoliths in a capillary, and its application to the trace determination of pesticides. Microchimica Acta, 182, 1775.

http://dx.doi.org/10.1007/s00604-015-1514-8 